\title{
Genotype-phenotype correlations in recessive RYR1-related myopathies
}

Kimberly Amburgey ${ }^{1}$, Angela Bailey ${ }^{1}$, Jean H Hwang ${ }^{2}$, Mark A Tarnopolsky ${ }^{3}$, Carsten G Bonnemann ${ }^{4}$, Livija Medne ${ }^{5}$, Katherine D Mathews ${ }^{6}$, James Collins ${ }^{7}$, Jasper R Daube ${ }^{8}$, Gregory P Wellman ${ }^{9}$, Brian Callaghan ${ }^{10}$, Nigel F Clarke ${ }^{2,11^{*}}$ and James J Dowling ${ }^{1 *}$

\begin{abstract}
Background: RYR1 mutations are typically associated with core myopathies and are the most common overall cause of congenital myopathy. Dominant mutations are most often associated with central core disease and malignant hyperthermia, and genotype-phenotype patterns have emerged from the study of these mutations that have contributed to the understanding of disease pathogenesis. The recent availability of genetic testing for the entire RYR1 coding sequence has led to a dramatic expansion in the identification of recessive mutations in core myopathies and other congenital myopathies. To date, no clear patterns have been identified in these recessive mutations, though no systematic examination has yet been performed.
\end{abstract}

Methods: In this study, we investigated genotype-phenotype correlations in a large combined cohort of unpublished $(n=14)$ and previously reported $(n=92)$ recessive $R Y R 1$ cases.

Results: Overall examination of this cohort revealed nearly $50 \%$ of cases to be non-core myopathy related. Our most significant finding was that hypomorphic mutations (mutations expected to diminish RyR1 expression) were enriched in patients with severe clinical phenotypes. We also determined that hypomorphic mutations were more likely to be encountered in non-central core myopathies. With analysis of the location of non-hypomorphic mutations, we found that missense mutations were generally enriched in the MH/CCD hotspots and specifically enriched in the selectivity filter of the channel pore.

Conclusions: These results support a hypothesis that loss of protein function is a key predictive disease parameter. In addition, they suggest that decreased RyR1 expression may dictate non-core related pathology though, data on protein expression was limited and should be confirmed in a larger cohort. Lastly, the results implicate abnormal ion conductance through the channel pore in the pathogenesis in recessive core myopathies. Overall, our findings represent a comprehensive analysis of genotype-phenotype associations in recessive RYR1-myopathies.

Keywords: Genotype-phenotype relationships, RYR1, Congenital myopathies

\footnotetext{
* Correspondence: nigel.clarke@health.nsw.gov.au; jamedowl@med.umich.edu

${ }^{2}$ Institute for Neuroscience and Muscle Research, Children's Hospital at

Westmead, University of Sydney, Level 3, Research Building Locked Bag 4001, Westmead, Sydney, NSW 2145, Australia

'Department of Pediatrics, Taubman Medical Research Institute, University of Michigan Medical Center, 5019 A. Alfred Taubman Biomedical Science

Research Building, 109 Zina Pitcher Place, Ann Arbor, MI 48109-2200, USA

Full list of author information is available at the end of the article
} 


\section{Background}

RyR1 is a skeletal muscle calcium release channel associated with excitation-contraction coupling [1]. The RYR1 gene is composed of 106 exons and encodes 5,038 amino acids, making it one of the largest genes in the human genome [2]. Mutations in RYR1 are the most common cause of congenital myopathies [3]. Both dominant and recessive mutations have been reported in RYR1. Dominant mutations have traditionally been associated with central core disease (CCD) and/or a susceptibility to malignant hyperthermia (MHS) [2], while recessive mutations predominate in patients with multiminicore disease $(\mathrm{MmD})$, centronuclear myopathy (CNM), and congenital fiber type disproportion (CFTD) [4-6]. At this time, no specific treatments are available for any RYR1-related myopathy, though modifying oxidative stress may be one therapeutic avenue [7].

Until recently, the majority of research on $R Y R 1$-related myopathies has focused on dominant mutations in $R Y R 1$ that lead to CCD and MHS phenotypes. Dominant mutations are enriched in three hotspots, with mutations in the $\mathrm{N}$-terminus and central regions most commonly associated with MHS and mutations in the C-terminus associated with CCD [8]. Previous literature may be biased due to the fact that analysis was limited to the hotspot regions. Comprehensive studies of selected dominant mutations have led to the hypothesis that MHS associated mutations cause RyR1 hyper-excitability, while CCD associated mutations result in chronic channel dysfunction, either through excitation-contraction uncoupling or by persistent channel leakiness [9].

Much less is known about recessive mutations and their mechanism(s) of disease. Several case series have been published reporting patients with recessive mutations, though overall they have lacked sufficient patient number and power needed for more broad conclusions. The largest existing study was performed by Klein and colleagues (2012), which included 36 families with recessive inheritance. They found, as compared to patients with dominant mutations, that patients with recessive $R Y R 1$ mutations had (1) more severe presentations with earlier onset, (2) more significant widespread weakness, and (3) more involvement of the extraocular and bulbar musculature. A smaller study from Zhou and colleagues (2007) observed that recessive $R Y R 1$ mutations are located throughout the gene and are associated with variable histological patterns and symptoms. An additional finding, from this and from other existing studies, is that many recessive $R Y R 1$ mutations are hypomorphic sequence changes that lead to markedly reduced or absent protein expression $[1,10]$.

Given the growing number of cases reported with recessive RYR1 mutations, a larger study combining and comparing these many reports is required in order to understand how various recessive mutations influence clinical phenotype, disease severity, and long term prognosis. The current study seeks to address this need by examining genotype-phenotype correlations in a cohort of 106 patients with recessive RYR1 mutations. This cohort includes 14 previously unreported cases together with published cases from the medical literature $(\mathrm{n}=$ 92). We specifically analyzed whether associations exist between mutation type and location, histopathologic diagnosis, and severity of clinical features. In addition, we analyzed the distribution of recessive mutations in relation to specific domains throughout the RyR1 protein. Overall, several associations were identified, including an association between the presence of a hypomorphic allele and increased clinical severity, association of the diagnosis of CNM and/or hypomorphic alleles with ophthalmoparesis, and an enrichment of missense mutations in the $\mathrm{MH} / \mathrm{CCD}$ hotspots and the pore selectivity filter. In all, this study provides a comprehensive analysis of genotype-phenotype relationships for recessive $R Y R 1$ mutations.

\section{Methods}

\section{Approvals}

For the previously unreported cases, all relevant information (clinical, diagnostic, etc.) and biologic samples were obtained using a protocol approved by the IRB at the University of Michigan.

\section{New patient ascertainment}

Clinical and diagnostic data from previously unreported cases were gathered from clinical records and from an online survey that was sent directly to colleagues or that accompanied RYR1 genetic testing results from PreventionGenetics. Information on recessive and nonclassical dominant cases (i.e. cases with dominant inheritance, but variability of symptoms due to reduced penetrance, monoallelic expression, etc.) was requested.

\section{RYR1 gene sequencing}

RYR1 sequencing of all coding regions was performed by PreventionGenetics using standard Sanger sequencing methods from patient genomic DNA. When possible, parental studies were performed to confirm inheritance.

\section{Protein expression}

Levels of RyR1 protein expression were assessed by western blot analysis of frozen muscle tissue (when available) sent from participating physicians using previously published methodology [11].

\section{Literature review}

To identify the known published cases of recessive RYR1related myopathy, we searched the medical literature for 
all previously reported recessive $R Y R 1$ cases and collated the clinical, histological and genetic information reported. A list of the references can be found at the end of the manuscript [1,2,4-6,9,10,12-33] and in Additional file 1: Table S1.

\section{Basis for genotype-phenotype assessment}

We compiled the following information: RYR1 mutations, parental testing results, family history, features of muscle biopsy, RyR1 protein expression, clinical features, age of onset, severity of weakness, motor function, respiratory function, and clinical/pathologic diagnosis. Cases with insufficient clinical or diagnostic information were excluded from the subsequent group analyses. Cases were clustered into core myopathies (MmCD, CCD, and other core myopathies), CNM and CNM-like myopathies, CFTD and other patterns. See Additional file 2: Table S2 for full descriptions.

\section{Disease severity ratings}

A disease severity rating scale (Additional file 3: Table S3) was created for this study to investigate whether there are relationships between mutation type/position, presence of ophthalmoparesis, histopathologic diagnosis and disease severity. Ambulatory status and respiratory status were used in this rating scale.

\section{Stratification of recessive RYR1 mutations}

Recessive mutations were divided into two groups based on in-silico analysis. In the first group, we included all mutations that were predicted to abolish or markedly decrease RyR1 protein production as hypomorphic alleles. These included nonsense mutations, frame-shift mutations, and splice mutations that lead to, or are predicted to cause, reduced levels of the mRNA transcript. The second group of recessive mutations included missense and small in-frame indels (insertions/deletions) which would likely result in approximately full-length but functionally abnormal RyR1 protein.

\section{Analysis of recessive RYR1 mutations}

We initially compared mutation type (missense/indels vs. hypomorphic alleles) with level of RyR1 protein expression (when available), histological diagnosis, clinical severity, and whether or not ophthalmoplegia was present using Chi-squared or Fisher's Exact tests. We then investigated whether the position of missense and small in-frame indel mutations (i.e. mutations that are likely to be incorporated into expressed RyR1 channels) correlates with clinical or histological features. We excluded hypomorphic mutations from this analysis since the position of these mutations in the RYR1 gene sequence is predicted to have little relationship with RyR1 function domains. Information about amino acid sequences that contribute to functional domains in RYR1 was obtained from our recent review of the literature [34]. For a list of functional domains and their amino acid sequences please see Additional file 4: Table S4. For the analysis of functional domains, recurrent mutations that occur in multiple individuals or families were only counted once (the most frequent clinical or histological phenotype associated with each mutation was used) to avoid skewing the results. The observed percentages of mutations residing in each domain and their 95\% or 99\% Wilson confidence intervals were calculated using SAS 9.2 software.

\section{Results}

A newly identified cohort of 29 families with RYR1-related myopathies

We identified 29 new cases (14 with recessive inheritance from 12 families and 15 with dominant inheritance from 14 families) with RYR1 mutations (Additional file 5: Table S5, Additional file 6: Table S6, Additional file 7: Table S7 and Additional file 8: Table S8). Thirteen were diagnosed with CCD, 7 with MmD, 8 with non-specific histological features (classified as RYR1-related myopathy or RRM), and 1 with congenital muscular dystrophy. Onset ranged from birth to adulthood. The majority of patients with recessive disease were non-ambulatory (10/14), while most patients with dominant inheritance were ambulatory $(11 / 15)$. Age at clinical review was not ascertained in the dataset and, therefore, may be a confounder for this result. Four of the patients (3 recessive, 1 dominant) required ventilatory support. Detailed clinical and pathologic information is included in Additional file 5: Table S5, Additional file 6: Table S6, Additional file 7: Table S7 and Additional file 8: Table S8.

There were 14 mutations found in the 14 dominant families (Additional file 7: Table S7). 7 (all missense) had been previously reported [8,35-39]. In terms of the novel variants, 4 are missense changes that are predicted (based on proximity to known mutations within the gene, on PolyPhen prediction, and on inheritance pattern) to be pathogenic/deleterious. One variant (patient $\mathrm{N}$ ) is a splice site change that is presumed to be pathogenic because it segregates with disease in the family. The variant in patient $\mathrm{T}$ is predicted to alter splicing and result in an inframe deletion of 25 amino acids. This variant is found in multiple affected family members.

There were 25 total sequence variants found in the 12 recessive families (Additional file 5: Table S5). 4 of the variants ( 1 nonsense and 3 missense) have been previously reported [12,31], while 21 were novel. 6 of the 21 novel mutations are predicted to result in the introduction of a premature stop codon. $13 / 15$ of the remaining variants are missense changes, and there is one single amino acid deletion and one duplication. The missense changes were 
presumed to be pathogenic based on several factors, including clinical context, mutation inheritance, and PolyPhen prediction. Only the recessive mutations were included in the subsequent genotype-phenotype analyses.

\section{Recessive RYR1 mutation cohort: histopathologic subtypes and mutation association}

A total of 106 cases with recessive RYR1 mutations were further analyzed. These included the 14 new cases mentioned above and 92 cases identified in the medical literature (Additional file 1: Table S1, which includes the specific references for the previously published cases). The histopathologic diagnoses for all cases are summarized in Figure 1. The most highly represented were the core myopathies (51\%) (inclusive of $\mathrm{MmD}$, CCD, atypical-core myopathy, and core/rod disease), followed by CNM/CNM-like myopathies (23.6\%).

We first examined the relationship between mutation type and histopathologic diagnosis, and several patterns emerged from this analysis (Figure 2A). Among patients with $\mathrm{MmD}$ and atypical core myopathies, there were

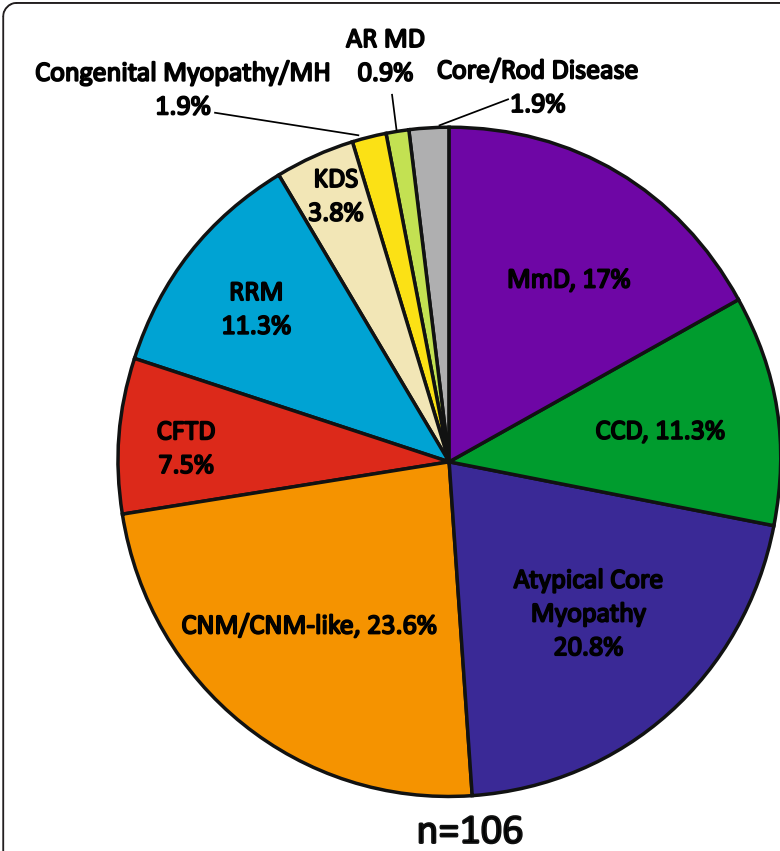

Figure 1 Breakdown of hispathologic diagnosis. Percentage breakdown of cases in the combined cohort by their predominant histopathologic pattern on muscle biopsy. Exceptions included are King Denborough Syndrome (KDS), which is a clinical diagnosis based primarily on specific dysmorphic features, and congenital myopathy plus malignant hyperthermia $(\mathrm{MH})$, where individuals had non-specific biopsy features plus a history of $\mathrm{MH}$. Abbreviations: multimincore disease (MmD), central core disease (CCD), centronuclear myopathy (CNM), congenital fiber type disproportion (CFTD), RYR1-related myopathy (RRM), autosomal recessive muscular dystrophy (AR MD). approximately equal percentages of patients with two non-hypomorphic (missense or in-frame indel) mutations and patients with at least one hypomorphic allele. On the other hand, the majority of patients with CCD had two non-hypomorphic mutations. The majority of patients with CNM/CNM-like, RRM and CFTD had at least one hypomorphic allele, although patient numbers were small for many of these subgroups.

\section{Mutation type, protein expression and clinical severity}

Mutation type was next compared with clinical severity (Figure 2B) using a severity scale based on ambulation and respiratory status (Additional file 3: Table S3). A significant association was identified between mutation type and clinical severity: a larger proportion (83\%) of patients with a severe phenotype (score $\geq 5$ on 8 point scale) had at least one hypomorphic allele compared to patients with a mild phenotype (score of $\leq 4)(51 \%)(p=0.0043)$. Another important factor on disease severity is the effect of the mutation on protein expression. There was a trend for low RyR1 protein levels (measured by western blot from patient biopsies) to be associated with a severe phenotype (Table 1), although data was available on relatively few biopsies $(\mathrm{n}=14)$ and was not statistically significant $(\mathrm{p}=$ 0.14). Conversely, patients with only missense or small inframe indel mutations (and no hypomorphic mutations) were significantly more likely to have a mild clinical phenotype. Of note, the likelihood of a severe phenotype was similar for all histological patterns. One bias to this observation is that age was not included in this analysis. Severity may be overestimated in patients too young to achieve certain motor milestones or may be overestimated in some patients as symptomology may improve with age.

\section{Mutation location, histopathologic subtype, and clinical severity}

Non-hypomorphic alleles were present throughout the gene (Figure 3), and further analysis was needed to identify associations with mutation type, histopathologic subtype and clinical severity. In total, 102 different non-hypomorphic mutations were present in the 106 recessive $R Y R 1$ patients we ascertained. We mapped the positions of these mutations to the known functional domains of RyR1. Analysis of the full cohort of nonhypomorphic mutations showed enrichment of mutations overall in $\mathrm{MH} / \mathrm{CCD}$ hotspot regions (52\% compared to an expected percentage based on RyR1 size of $37.8 \%$, 99\% confidence interval of 39-54\%), though no significant enrichment in any one specific hotspot domain. We next examined more specifically whether there is an association between the position of mutations and either histological diagnosis (Table 2) or clinical severity (Table 3). Of note, by visual inspection there appears to be additional regions in $R Y R 1$ where there are clusters of non-hypomorphic 

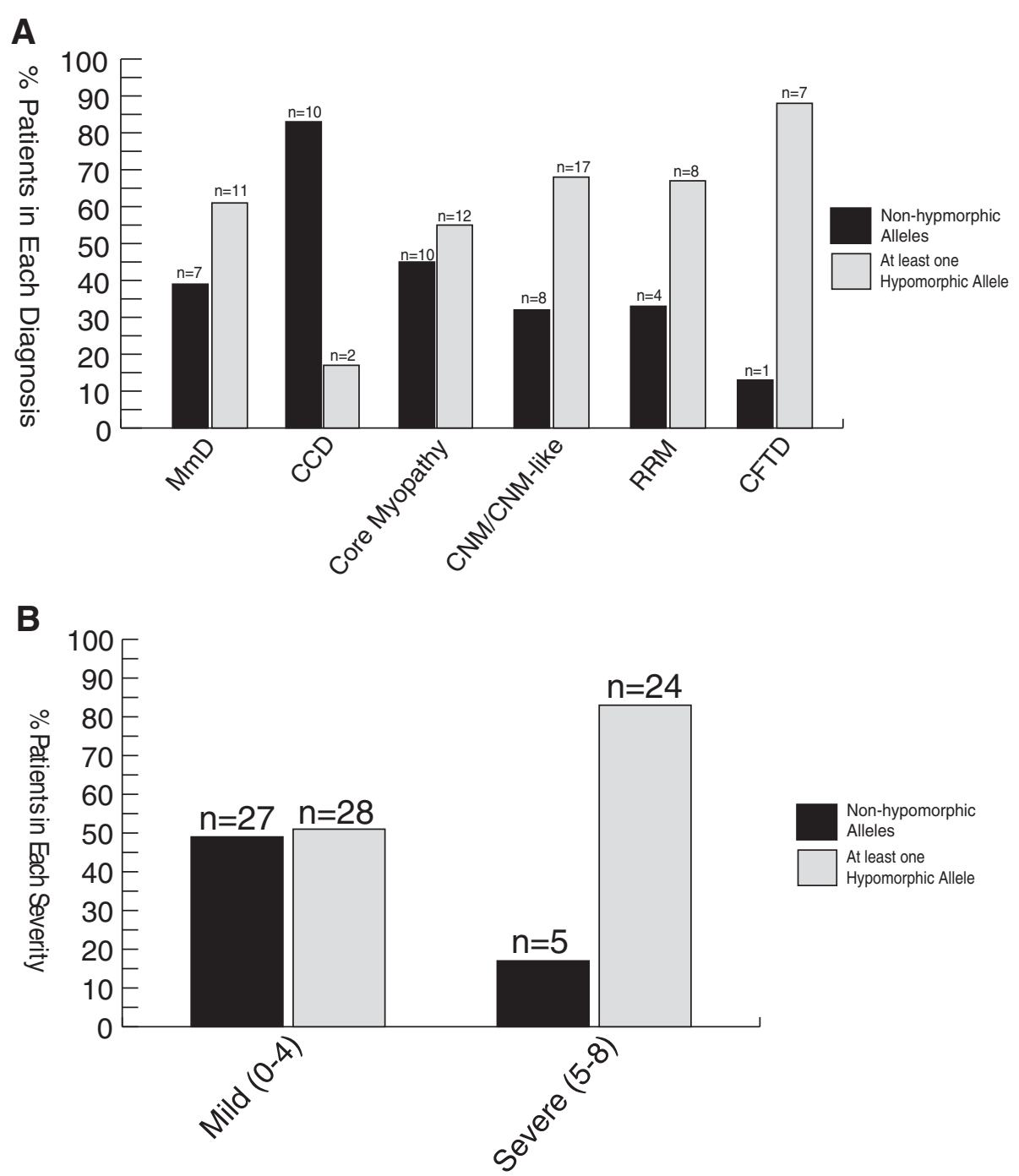

Figure 2 Assessment of associations with mutation type. (A) Mutation types among histopalogic diagnosis. Abbreviations: multimincore disease (MmD), central core disease (CCD), centronuclear myopathy (CNM), RYR1-related myopathy (RRM), congenital fiber type disproportion (CFTD). (B) Mutation types among severity groups. Clinical severity scores of 0-4 were considered mild, while scores of 5-8 were considered severe based on the criteria listed in Additional file 3: Table S3.

alleles. Because these clusters fell outside of any known functional region, we did not analyze them further.

Subgroup analysis for histological diagnosis showed an association most strongly for CCD, particularly in $\mathrm{MH} /$ CCD hotspot region 3 (Table 2), a C-terminal region that spans residues 3916-4942. 59\% of non-hypomorphic CCD mutations were located in hotspot region 3 , a significant increase (99\% confidence interval between 30-83\%) compared to an expected percentage (based on its size compared to the overall size of RyR 1 ) of only $20.4 \%$. The selectivity filter of the channel pore (Gly4891-D4900) which sits within the triadin-binding region and MH/CCD hotspot 3, was also significantly enriched for mutations in CCD patients (18\% of mutations compared to $0.002 \%$ expected by size, with $99 \%$ confidence interval of $5-49 \%$ ).
Subgroup analysis identified other possible associations with histopathologic diagnosis as well. The DHPR-binding domains had fewer mutations than expected in the full mutation cohort and CCD subgroup analysis (Table 2). Mutations associated with non-specific histological patterns clustered more than expected in MH/CCD hotspot regions 2. However, no associations with mutation position were seen for $\mathrm{MmD}, \mathrm{CNM}$ or CFTD.

In terms of clinical severity, two observations emerged from the analysis of non-hypomorphic mutations. These were that mutations in MH/CCD hotspot 3 (58\% association, $\mathrm{n}=19$ mutations) and in the triadin binding domain (100\% association, though for $\mathrm{n}=3$ ), which sits within $\mathrm{MH} / \mathrm{CCD}$ hotspot 3, were associated with a severe phenotype more often than expected by chance and than with 
Table 1 RYR1 protein level and clinical severity

\begin{tabular}{ccc}
\hline Patient identifier & $\begin{array}{c}\text { RyR1 protein level } \\
\text { (\% of normal) } \\
\text { Mild 0-4 }\end{array}$ & Severity rating \\
\hline 21 & $40+/-6$ & 0 \\
167 & 44 & 1 \\
178 & $44+/-4$ & 1 \\
S & 55 & 1 \\
168 & 16 & 2 \\
37 & $51+/-7$ & 3 \\
54 & $22+/-12$ & 4 \\
\hline & Severe 5-8 & 5 \\
\hline 179 & $14+/-3$ & 6 \\
55 & $15+/-8$ & 6 \\
123 & 10 & 7 \\
132 & $38+/-4$ & 8 \\
\hline
\end{tabular}

RyR1 protein levels are presented as \% of normal $+/-$ standard error of the mean. Clinical severity scores of 0-4 were considered mild, while scores of 5-8 were considered severe based on the criteria listed in Additional file 3: Table S3.

the overall cohort (31.3\%) (Table 3). Of note, we only were able to derive severity scores for 67 of the non-hypomorphic mutations, which thus limited the scope of this particular analysis.

\section{Mutation type/location and ophthalmoparesis}

In terms of specific clinical symptoms, ophthalmoparesis is a key clinical feature found in a subset of patients with RYR1 mutations. In some contexts (e.g. minicore myopathy) it can be used to help distinguish patients with RYR1 mutations from other genetic subtypes [40]. We examined for an association between ophthalmoparesis and histopathological subtype, mutation type and clinical severity. Several associations were identified (Figure 4). Among all histopathological subtypes, ophthalmoparesis was more likely to be associated with a diagnosis of CNM/CNM-like ( $\mathrm{p}=$ 0.006). Additionally, a larger proportion of patients with at least one hypomorphic allele (72\%) had ophthalmoparesis than those with non-hypomorphic mutations (32\%) ( $\mathrm{p}=$ 0.0003). Conversely, there was no significant association between ophthalmoparesis and clinical severity (data not shown) or between ophthalmoparesis and mutation position (Table 3).

\section{Discussion}

There has been a recent explosion in the identification of new cases of congenital myopathies due to RYR1 mutations, particularly those with recessive inheritance. It is thus now possible to perform mutation correlations that are adequately powered to uncover significant associations. In this study, we present a comprehensive genotype-phenotype analysis of $R Y R 1$ mutations from more than 100 cases of recessively inherited RYR1related myopathies. This study represents the largest examination of recessive cases to date, and includes 14 new cases plus analysis of all recessive cases in the medical literature that we could ascertain. Using this cohort, we have identified several statistically significant associations that provide clues about disease mechanisms in different histological diagnoses and about important

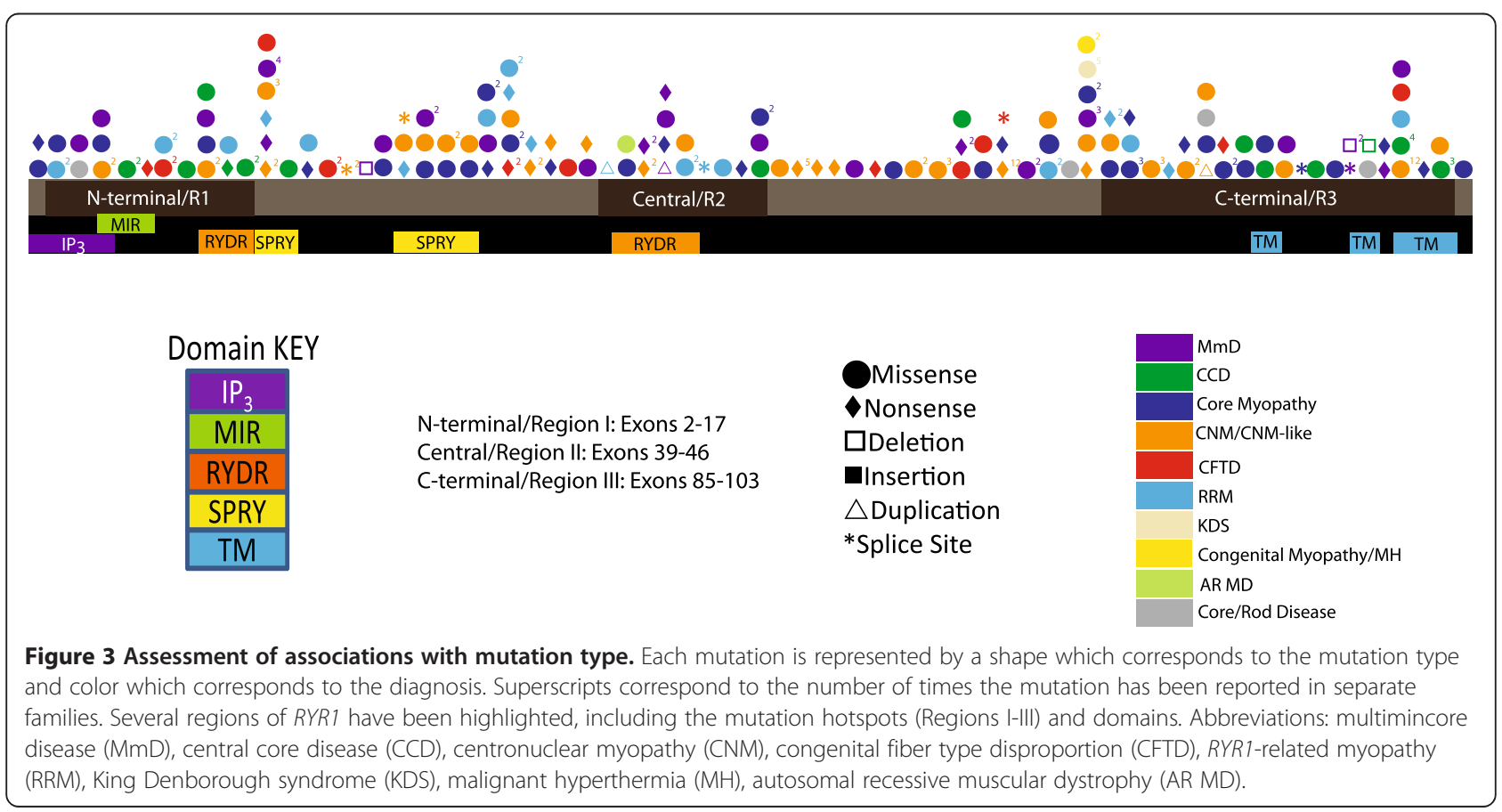


Table 2 Proportions of recessive non-hypomorphic missense mutations in known functional RYR1 domains analyzed by histological diagnosis

\begin{tabular}{|c|c|c|c|c|c|c|c|c|}
\hline \multirow[b]{3}{*}{ Domains } & \multirow[b]{3}{*}{ Expected $\% \wedge$} & \multicolumn{7}{|c|}{ Diagnostic categories } \\
\hline & & $\begin{array}{l}\text { All mutations }{ }^{+} \\
\quad(\mathrm{N}=102)\end{array}$ & $\begin{array}{c}\text { CCD } \\
(\mathrm{N}=17)\end{array}$ & $\begin{array}{c}\mathrm{MmD} \\
(\mathrm{N}=16)\end{array}$ & $\begin{array}{l}\text { Atypical core } \\
\text { myopathy } \\
(\mathrm{N}=27)\end{array}$ & $\begin{array}{c}\text { CNM/CNM- } \\
\text { like myopathy } \\
(N=21)\end{array}$ & $\begin{array}{l}\text { CFTD } \\
(\mathrm{N}=5)\end{array}$ & $\begin{array}{l}\text { RYR1-related } \\
\text { myopathy } \\
(\mathrm{N}=10)\end{array}$ \\
\hline & & Observed \% (Cl) & Observed \% (Cl) & Observed \% (Cl) & Observed \% (Cl) & Observed \% (Cl) & Observed \% (Cl) & Observed \% (Cl) \\
\hline $\mathrm{MH} / \mathrm{CCD}$ hotspot domains & 37.8 & $52(39-64) * *$ & $88(53-98) * *$ & $38(19-61)$ & $52(34-69)$ & $43(25-64)$ & $0(0-40)$ & $60(31-83)$ \\
\hline Hotspot domain 1 & 11.5 & $16(10-24)$ & $24(9-47)$ & $13(4-36)$ & $11(4-28)$ & $5(1-22)$ & $0(0-40)$ & $30(11-60)$ \\
\hline Hotspot domain 2 & 5.9 & $10(5-17)$ & $6(1-27)$ & $13(4-36)$ & $11(4-28)$ & $5(1-22)$ & $0(0-40)$ & $30(8-68)^{* *}$ \\
\hline Hotspot domain 3 & 20.4 & $26(19-36)$ & $59(30-83) * *$ & $13(4-36)$ & $30(16-49)$ & $33(17-55)$ & $0(0-40)$ & $0(0-28)$ \\
\hline Selectivity filter ${ }^{\circ}$ & $0.002 \%$ & $3(0.7-11)^{* *}$ & $18(5-49)^{* *}$ & $0(0-20)$ & $0(0-13)$ & $0(0-16)$ & $0(0-43)$ & $0(0-28)$ \\
\hline Triadin & 1.2 & $6(2-15) * *$ & $18(5-49) * * a$ & $6(1-28)$ & $4(1-18)$ & $5(1-22)$ & $0(0-43)$ & $0(0-28)$ \\
\hline DHPR & 31.4 & $22(15-31)$ & $6(0-27)$ & $19(7-43)$ & $19(8-37)$ & $19(8-40)$ & $20(4-62)$ & $60(31-83)$ \\
\hline SPRY domains & 8.0 & $10(5-17)$ & $6(1-27)$ & $6(1-28)$ & $0(0-13)$ & $14(5-35)$ & $20(4-62)$ & $10(2-40)$ \\
\hline S100A1 & 12.0 & $5(2-11)$ & $6(1-27)$ & $6(1-28)$ & $4(1-18)$ & $0(0-16)$ & $20(4-62)$ & $10(2-40)$ \\
\hline apoCaM & 11.0 & $7(3-14)$ & $0(0-18)$ & $0(0-20)$ & $15(6-33)$ & $14(4-35)$ & $0(0-43)$ & $0(0-28)$ \\
\hline $\mathrm{CaCaM}$ & 2.7 & $1(0-5)$ & $0(0-18)$ & $0(0-20)$ & $4(1-18)$ & $0(0-16)$ & $0(0-43)$ & $0(0-28)$ \\
\hline Interdomain interactions & 1.5 & $1(0-5)$ & $0(0-18)$ & $0(0-20)$ & $0(0-13)$ & $5(1-22)$ & $0(0-43)$ & $0(0-28)$ \\
\hline
\end{tabular}

${ }^{+}$Six mutations are only included in the 'All mutations' analysis as histological information was not available for further classification. $\wedge$ Expected percentage (\%) $=$ the percentage of mutations in each domain that would occur by chance alone, based on the size of the domain relative to the full RyR1 amino acid sequence. Observed \%= percentage of mutations in each functional domain. All Wilson confidence intervals (CI) denote $95 \%$ intervals unless indicated by ${ }^{*}$, which are $99 \%$ confidence intervals. Statistically significant results are shown in bold font. $\mathrm{N}=$ number of mutations in each diagnostic category. ${ }^{\circ} \mathrm{Amino}$ acids $4891-4900$. ${ }^{a}$ All CCD mutations in the triadin-binding region also lie in the selectivity filter of the pore (amino acids 4895-4900) and so are likely to have their main effects on ion conductivity directly, rather than on triadin binding. $\mathrm{CCD}=$ central core disease. $\mathrm{MmD}=$ multi-minicore disease. $\mathrm{CFTD}=$ congenital fiber type disproportion. $\mathrm{MH}=$ malignant hyperthermia. $\mathrm{DHPR}=$ dihydropyridine receptor. apoCaM $=$ calmodulin without $\mathrm{Ca}{ }^{2+}$

bound. $\mathrm{CaCaM}=$ calmodulin with $\mathrm{Ca}^{2+}$ bound. 
Table 3 An analysis of the position of all recessive non-hypomorphic RYR1 mutations vs severity \& ophthalmoparesis

\begin{tabular}{|c|c|c|c|c|}
\hline & $\begin{array}{l}\text { Mutations associated } \\
\text { with mild phenotypes/ } \\
\text { total cohort }\end{array}$ & $\begin{array}{c}\text { Percentage of mutations } \\
\text { with mild } \\
\text { phenotypes (Cl) }\end{array}$ & $\begin{array}{c}\text { Mutations associated } \\
\text { with ophthalmoplegia/ } \\
\text { Total mutations }\end{array}$ & $\begin{array}{l}\text { Percentage of mutations } \\
\text { associated with } \\
\text { ophthalmoplegia (Cl) }\end{array}$ \\
\hline Total cohort* & $46 / 67$ & 68.7 & $43 / 102$ & 42.2 \\
\hline \multicolumn{5}{|l|}{ Domain } \\
\hline $\begin{array}{l}\text { Combined } \mathrm{MH} / \mathrm{CCD} \\
\text { hotspot domains }\end{array}$ & $21 / 35$ & $60(44-74)$ & $17 / 52$ & $33(21-46)$ \\
\hline Hotspot domain 1 & $9 / 12$ & $75(46-92)$ & $6 / 16$ & $38(18-61)$ \\
\hline Hotspot domain 2 & $4 / 4$ & $100(54-100)$ & $2 / 10$ & $20(5-52)$ \\
\hline Hotspot domain 3 & $8 / 19$ & $42(23-64)$ & $9 / 26$ & $35(19-54)$ \\
\hline Interdomain interactions & $1 / 1$ & $100(22-100)$ & $1 / 1$ & $100(22-100)$ \\
\hline Triadin & $0 / 3$ & $0(0-53)$ & $2 / 7$ & $29(8-65)$ \\
\hline DHPR & $9 / 10$ & $90(46-100)$ & $5 / 20$ & $25(11-47)$ \\
\hline S100A1 & $2 / 2$ & $100(22-100)$ & $1 / 5$ & $20(2-64)$ \\
\hline apoCaM & $4 / 4$ & $100(37-100)$ & $4 / 7$ & $57(25-84)$ \\
\hline $\mathrm{CaCaM}$ & $0 / 0$ & - & $0 / 1$ & $0(0-78)$ \\
\hline SPRY domains & $3 / 5$ & $60(17-92)$ & $7 / 11$ & $64(35-85)$ \\
\hline
\end{tabular}

Severity scores between 0 and 4 were classed as mild and scores between 5 and 8 were classed as severe (Additional file 3: Table S3). Two results in the analysis of disease severity vs mutation domain deviated significantly from expected ratios. Mutations in MH/CCD hotspot 3 and in the triadin binding domain (with sits within hotspot 3) were associated with a severe phenotype more often than expected by chance. There was no evidence that non-hypomorphic mutations in specific domains were associated with a greater or lower chance of ophthalmoparesis. ${ }^{*}$ To reduced bias, each non-hypomorphic recessive RYR1 mutation was counted only once, even if several individuals have been reported with the mutation. For the analysis of phenotype severity, severity scores were averaged when severity data was available on several individuals with a mutation. For the analysis of ophthalmoparesis, mutations were classified as associated with ophthalmoplaresis if any patient with the mutation was reported to have ophthalmoparesis. $\mathrm{Cl}=95 \%$ Wilson confidence intervals. Bold font shows where results are significantly different from expected ratios.

determinants of disease severity. The most notable finding is an association between increased disease severity and the presence of at least one hypomorphic (nonsense, frame shift, splice site) allele. Additional important observations include the identification of enrichment of missense mutations in specific locations associated with particular histopathologic subtypes of RYR1-related myopathy, suggesting that histological phenotype is at least partially determined by mutation position.

\section{General cohort statistics: core vs non-core myopathies}

Historically, the overwhelming majority of congenital myopathy patients with mutations in $R Y R 1$ had a core myopathy. The discovery of $R Y R 1$ mutations in cases of noncore myopathy is a relatively recent phenomenon, and the relative prevalence of such cases has been uncertain. We were surprised to find that $49 \%$ of recessive cases identified in our study were non-core myopathies. Once ascertainment bias is removed (i.e. a historical skewing toward RYR1 mutation identification in core myopathies because these were the first $R Y R 1$ myopathies identified), it is possible that non-core myopathies ultimately account for more than $50 \%$ of recessive RYR1 disease. However, a potential caveat to this assertion is a reverse bias created by the fact that some patients with core myopathy (particularly central core disease) are assumed to have RYR1 mutations and thus not confirmed at the genetic level.
Mutation type and clinical severity: hypomorphic alleles are associated with severe disease

One of our primary goals was to see whether disease severity could be predicted based on the type of mutation. We found a clear, statistically significant association between the presence of a hypomorphic mutation and a severe clinical picture. This data is in keeping with analyses of smaller patient cohorts, and suggests that reduced total RyR1 protein levels is an important disease mechanism that heralds more severe disease [22]. Of note, no patient has yet been reported with two fully hypomorphic RYR1 mutations, suggesting that some residual RyR1 function is required for life. This is in keeping with the perinatal lethal phenotype of Ryr 1 knockout mice [41]. An extrapolation of this hypothesis is that all recessive missense/indel mutations that are found in association with a hypomorphic mutation or that are present in a homozygous state are unlikely to completely abolish RyR1 function. One can also predict that increasing expression of RyR1, even of RyR1 subunits with abnormal characteristics due to missense or other mutations, may serve to ameliorate disease. Additional research will be necessary to test this hypothesis.

\section{Mutations, diagnosis, and functional domains}

We also sought to identify patterns between mutation type/location and specific histopathologic diagnoses as 

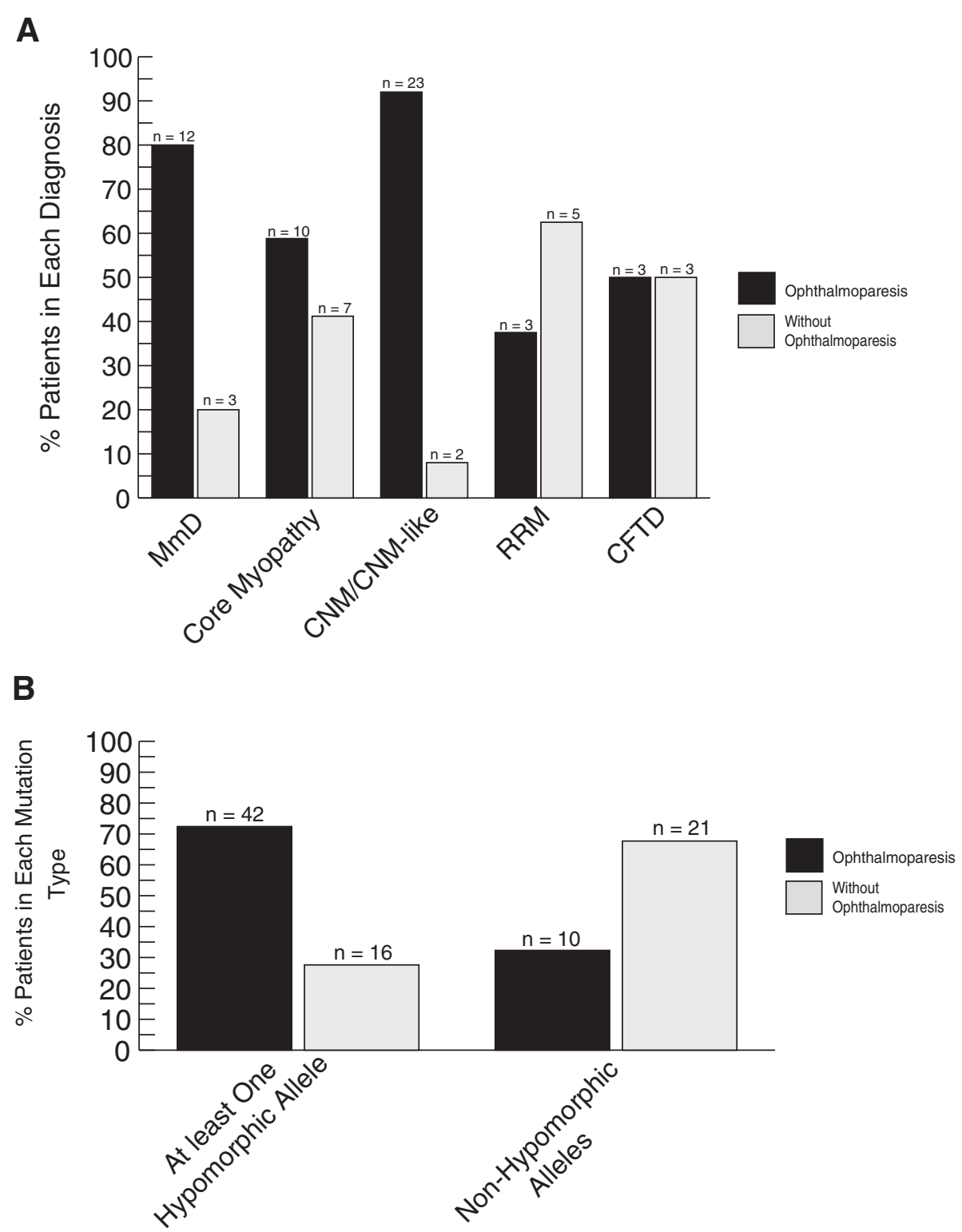

Figure 4 Assessment of associations with ophthalmoparesis. (A) Ophthalmoparesis among hispathologic diagnosis. Abbreviations: multimincore disease (MmD), centronuclear myopathy (CNM), RYR1-related myopathy (RRM), congenital fiber type disproportion (CFTD). (B) Ophthalmoparesis among mutation types.

they may give insights into the pathogenesis of the different histological patterns that can arise. One significant trend identified was that hypomorphic mutations were more likely to be seen in non-CCD myopathy subtypes compared to central core disease. This suggests that non-central core pathology is, in part, dictated by reduction in RyR1 expression and presumably reduced RyR1 function. This would be consistent with animal model data, particularly from the zebrafish model of RYR1-myopathy, which has severely reduced expression ( $>90 \%$ reduction), obvious myopathic features, but no central cores and very few true minicores $[7,42]$. One explanation may be that CCD is often due (particularly in de novo/dominant causes) to mutations that result in the expression of RyR1 channels with abnormal properties while all other histological phenotypes are associated with a generalized reduction in RyR1 function.

We also identified several interesting associations between clinical/histological phenotypes and mutation location. Previous studies have found no patterns in the location of recessive mutations, and a prevailing assumption has been that recessive mutations are randomly distributed throughout the gene [1]. However, our analysis reveals that there are significant enrichments of 
mutations in known functional domains of the protein and in association with particular diagnoses. In particular, we detected an over-representation of recessive nonhypomorphic RYR1 mutations in the combined $\mathrm{MH} / \mathrm{CCD}$ hotspots and in the subgroup analysis there was a strong association between CCD mutations and hotspot region 3 that exceeded $99 \%$ confidence intervals. This is the same pattern that is seen for dominant CCD mutations, which are more often in hotspot region 3 than regions 1 or 2 [8] and suggests that dominant and recessive forms of CCD may share similar disease mechanisms.

One association that provides a clue about disease pathogenesis is the enrichment of recessive missense mutations in the selectivity filter of the channel pore, a 10 amino acid motif that conserved in all RyRs and that is critical for channel function [43]. This region only occupies $0.002 \%$ of the linear protein sequence, but accounts for $3 \%$ of recessive non-hypomorphic mutations overall and almost $20 \%$ of recessive CCD mutations. This implicates abnormalities in ion conductance or ion selectivity in the pathogenesis of recessive $C C D$, a mechanism that has been linked to dominant CCD mutations (reviewed in $[9,27])$. The enrichment of recessive missense mutations in the triadin-binding domain is due to the fact that this domain contains the selectivity filter. The finding that fewer mutations than expected were located in DHPR binding regions may indicate that interactions with these RyR1-binding proteins tend not to be important in the pathogenesis of the known RYR1 myopathies. Some associations are expected by chance when making multiple comparisons and it would be ideal to replicate these findings in a second cohort of mutations as confirmation.

\section{Conclusions}

In all, we present a comprehensive genotype-phenotype study of recessive RYR1 mutations. The insights we provide reveal the utility of such a study, and show the first associations between mutation type and location and clinical severity and diagnosis. In particular, we highlight the $\mathrm{MH} / \mathrm{CCD}$ hotpspot regions, (and the selectivity filter that lies within hotspot region 3), as likely to be important in the pathogenesis of many recessive RYR1-related myopathies. The study of additional patients in the future will confirm and refine these associations.

\section{Additional files}

Additional file 1: Table S1. Literature review of recessive $R Y R 1$ mutations. References for all cases in the literature are included at the end of the spreadsheet and in the manuscript. No diagnosis refers to those cases where histologic information as not available to further define the diagnosis.

Additional file 2: Table S2. Grouping patients into broad diagnostic categories for analysis.
Additional file 3: Table S3. Criteria for severity scores. Ambulatory and respiratory ratings were added together to calculate the overall severity score Abbreviations: pulmonary function testing (PFT), ventilator (Vent), continuous positive airway pressure (CPAP), bilevel positive airway pressure (BiPAP).

Additional file 4: Table S4. Functional domains in the ryanodine receptor type1. *Amino acids are numbered relative to the full RyR1 amino acid sequence (NM_000540). DHPR = dihydropyridine receptor, apoCaM = calmodulin without bound $\mathrm{Ca} 2+, \mathrm{CaCaM}=$ calmodulin with bound Ca2+. Information summarized from Hwang et al., 2012.

Additional file 5: Table S5. Clinical characteristics of newly reported recessive RYR1 mutations. Severity scores based on criteria listed in Additional file 3: Table S3. Also included are the PolyPhen2 predictions for all novel missense mutations (Probably Damaging (PRD), Possibly Damaging (POD)) and the severity (scale 0-1, 0 indicating least severe, 1 indicating most severe). Origin of the mutation is designated ${ }^{\mathrm{M}}$ for maternal and ${ }^{\mathrm{P}}$ for paternal. For patients $F \& G$, carrier testing was only performed on the father. Since one mutation was identified, the other mutation is presumed to be maternal, which is denoted by ${ }^{M^{*}}$. Patients $O$ and $P$ (siblings) were presumed recessive based on clinical presentation, lack of parental symptoms, and the presence of two mutations. Parental testing was not performed, so it is formally possible (though unlikely) that the two mutations exist in cis. For patient $Y$, one mutation was identified as maternal in origin. Carrier testing of the father did not identify the second mutation, therefore, it is presumed to be de novo. Abbreviations: Patient ID $(\mathrm{ID})$, siblings (F\&G, O\&P) $\left(^{*}\right)$, diagnosis (DX), multimincore disease $(\mathrm{MmD})$, core myopathy (CM), RYR1-related myopathy (RRM), autosomal recessive muscular dystrophy (AR MD), central core disease (CCD), first year of life (FYOL), Weakness: proximal (P), distal (D), facial (F), neck (N); rigid spine (RS), ophthalmoparesis $(\mathrm{OPH})$, respiratory distress $(\mathrm{RD})$, ventilator (Vent), feeding difficulties (FD), malignant hyperthermia $(\mathrm{MH})$, creatine kinase (CK).

Previously reported mutations: ${ }^{a}$ Bevilacqua, et al., 2011, 'b Zhou, et al., 2010.

Additional file 6: Table S6. Hispathologic findings of newly reported recessive RYR1 mutations. Diagnosis of cases without a muscle biopsy was based on family history of the disease. Mutations previously reported in the medical literature ( ${ }^{B}$ Bevilacqua, et al., 2011, b $\mathrm{Zhou}$, et al., 2010). Origin of the mutation is designated ${ }^{\mathrm{M}}$ for maternal and ${ }^{\mathrm{P}}$ for paternal. For patients $F \& G$, carrier testing was only performed on the father. Since one mutation was identified, the other mutation is presumed to be maternal, which is denoted by ${ }^{\mathrm{M}^{*}}$. For patient $\mathrm{Y}$, one mutation was identified as maternal in origin. Carrier testing of the father did not identify the second mutation, therefore, it is de novo which is denoted by ${ }^{D}$. Abbreviations: Patient ID (ID), siblings (F\&G, O\&P) $\left(^{*}\right)$, diagnosis (DX), multimincore disease (MmD), core myopathy (CM), RYR1-related myopathy (RRM), autosomal recessive muscular dystrophy (AR MD), central core disease (CCD), central cores $(C C)$, minicores $(M C)$, internalized nuclei (IN), central nuclei (CN).

Additional file 7: Table S7. Clinical characteristics of newly reported dominant RYR1 mutations. Severity scores based on criteria listed in Additional file 3: Table S3. Polyphen2 scores are also included for all novel missense mutations (Probably Damaging (PRD)) and the severity (scale 0-1, 0 indicating least severe, 1 indicating most severe). $\wedge$ indicates that the silent mutation is predicted to create a new splice donor site resulting in a 25 amino acid in-frame deletion. Previously reported mutations: ${ }^{a}$ Lynch,

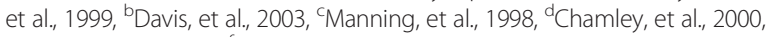
'Monnier, et al., 2001, 'Davis, et al., 2002). Origin of the mutation is designated ${ }^{\mathrm{M}}$ for maternal, ${ }^{\mathrm{P}}$ for paternal, or ${ }^{\mathrm{D}}$ for de novo. Abbreviations: Patient ID (ID), siblings (B\&C), diagnosis (DX), central core disease (CCD), RYR1-related myopathy (RRM), multimincore disease (MmD), first year of life (FYOL), Weakness: proximal (P), distal (D), facial (F), neck (N); rigid spine (RS), ophthalmoparesis $(\mathrm{OPH})$, respiratory distress $(\mathrm{RD})$, ventilator (Vent), feeding difficulties (FD), malignant hyperthermia $(\mathrm{MH})$, creatine kinase (CK).

Additional file 8: Table S8. Hispathologic findings of newly reported dominant RYR1 mutations. Previously reported mutations: 'Lynch, et al., 1999, 'Davis, et al., 2003, 'Manning, et al., 1998, 'Chamley, et al., 2000, 'Monnier, et al., 2001, 'Davis, et al., 2002) [8,36-39,44]. Origin of the mutation is designated ${ }^{M}$ for maternal, ${ }^{P}$ for paternal, or ${ }^{D}$ for de novo. Abbreviations: Patient ID (ID), siblings (B\&C), diagnosis (DX), central core disease (CCD), RYR1-related myopathy (RRM), multimincore disease (MmD), central cores $(C C)$, minicores $(M C)$, internalized nuclei $(\mathrm{IN})$, central nuclei $(C N)$. 


\section{Competing interests}

The authors declare that they have no competing interests. MT was paid consulting fees by Biomarin in relation to an unrelated clinical trial, and was paid by Genzyme/Sanofi for lecture and travel costs associated with the Steps Forward Meeting $(2009,2011,2012)$.

\section{Authors' contributions}

KA participated in the design and coordination of the study, assisted in data collection and interpretation, and drafted the manuscript. AB was responsible for data collection and interpretation. JH assisted with data management and helped to draft the manuscript. MT, CB, LM, DM, JC, JRD, and GW provided new cases of RYR1 myopathy and also provided critical reading of the manuscript. BC performed the statistical analysis. JJD and NC conceived the study, participated in data analysis, and drafted and edited the manuscript. All authors read and approved the final manuscript.

\section{Acknowledgements}

This work has been supported by a grant from the NIH (K08AR054835 to JJD) and the National Health \& Medical Research Council (NC; grants 1035828 \& 1022707). JH is also supported by the Albert Einstein College of Medicine Senior Research Fellowship. Additional support comes from the Taubman Research Institute Emerging Scholar fund. We thank Liz Barnes from the NHMRC Clinical Trials Centre at University of Sydney for the statistical analysis.

\section{Author details}

${ }^{1}$ Department of Pediatrics, Taubman Medical Research Institute, University of Michigan Medical Center, 5019 A. Alfred Taubman Biomedical Science Research Building, 109 Zina Pitcher Place, Ann Arbor, MI 48109-2200, USA. ${ }^{2}$ Institute for Neuroscience and Muscle Research, Children's Hospital at Westmead, University of Sydney, Level 3, Research Building Locked Bag 4001, Westmead, Sydney, NSW 2145, Australia. ${ }^{3}$ Department of Neuromuscular and Neurometabolic Disease, McMaster University Medical Center, Hamilton, Ontario, Canada. ${ }^{4}$ Neuromuscular and Neurogenetic Disorders of Childhood Section, National Institutes of Health, Bethesda, MD, USA. ${ }^{5}$ Division of Neurology, Children's Hospital of Philadelphia, Philadelphia, PA, USA. ${ }^{6}$ Departments of Pediatrics and Neurology, University of lowa, Carver College of Medicine, lowa City, IA, USA. ${ }^{7}$ Department of Pediatric Neurology, Cincinnati Children's Hospital Medical Center, Cincinnati, OH, USA ${ }^{8}$ Department of Neurology, Mayo Foundation for Medical Education and Research, Rochester, MN, USA. ${ }^{9}$ The Delta Pathology Group, Shreveport, LA, USA. ${ }^{10}$ Department of Neurology, University of Michigan Medical Center, Ann Arbor, MI, USA. ${ }^{11}$ Discipline of Paediatrics and Child Health, University of Sydney, Sydney, Australia.

\section{Received: 6 March 2013 Accepted: 1 August 2013}

Published: 6 August 2013

\section{References}

1. Zhou H, Jungbluth $H$, Sewry CA, Feng L, Bertini E, Bushby K, Straub V, Roper $H$, Rose MR, Brockington M, et al: Molecular mechanisms and phenotypic variation in RYR1-related congenital myopathies. Brain 2007, 130:2024-2036.

2. Robinson R, Carpenter D, Shaw MA, Halsall J, Hopkins P: Mutations in RYR1 in malignant hyperthermia and central core disease. Hum Mutat 2006, 27:977-989.

3. Amburgey K, McNamara N, Bennett LR, McCormick ME, Acsadi G, Dowling $\mathrm{JJ}$ : Prevalence of congenital myopathies in a representative pediatric united states population. Ann Neurol 2011, 70:662-665.

4. Jungbluth H: Multi-minicore Disease. Orphanet J Rare Dis 2007, 2:31.

5. Wilmshurst JM, Lillis S, Zhou H, Pillay K, Henderson H, Kress W, Muller CR, Ndondo A, Cloke V, Cullup T, et al: RYR1 mutations are a common cause of congenital myopathies with central nuclei. Ann Neurol 2010, 68:717-726

6. Clarke NF, Waddell LB, Cooper ST, Perry M, Smith RL, Kornberg AJ, Muntoni F, Lillis S, Straub V, Bushby K, et al: Recessive mutations in RYR1 are a common cause of congenital fiber type disproportion. Hum Mutat 2010, 31:E1544-E1550.

7. Dowling JJ, Arbogast S, Hur J, Nelson DD, McEvoy A, Waugh T, Marty I, Lunardi J, Brooks SV, Kuwada JY, Ferreiro A: Oxidative stress and successful antioxidant treatment in models of RYR1-related myopathy. Brain 2012, 135(Pt 4):1115-1127.
8. Davis MR, Haan E, Jungbluth H, Sewry C, North K, Muntoni F, Kuntzer T, Lamont $\mathrm{P}$, Bankier A, Tomlinson $\mathrm{P}$, et al: Principal mutation hotspot for central core disease and related myopathies in the C-terminal transmembrane region of the RYR1 gene. Neuromuscul Disord 2003, 13:151-157.

9. Treves $\mathrm{S}$, Jungbluth $\mathrm{H}$, Muntoni $\mathrm{F}$, Zorzato F: Congenital muscle disorders with cores: the ryanodine receptor calcium channel paradigm. Curr Opin Pharmacol 2008, 8:319-326.

10. Monnier N, Marty I, Faure J, Castiglioni C, Desnuelle C, Sacconi S, Estournet B, Ferreiro A, Romero N, Laquerriere A, et al: Null mutations causing depletion of the type 1 ryanodine receptor (RYR1) are commonly associated with recessive structural congenital myopathies with cores. Hum Mutat 2008, 29:670-678.

11. Anderson GL, Manson J, Wallace R, Lund B, Hall D, Davis S, Shumaker S, Wang CY, Stein E, Prentice RL: Implementation of the Women's Health Initiative study design. Ann Epidemiol 2003, 13:S5-S17.

12. Bevilacqua JA, Monnier N, Bitoun M, Eymard B, Ferreiro A, Monges S, Lubieniecki F, Taratuto AL, Laquerriere A, Claeys KG, et al: Recessive RYR1 mutations cause unusual congenital myopathy with prominent nuclear internalization and large areas of myofibrillar disorganization. Neuropathol Appl Neurobiol 2011, 37:271-284.

13. Carpenter $D$, Ismail $A$, Robinson RL, Ringrose $C$, Booms $P$, lles DE, Halsall PJ, Steele D, Shaw MA, Hopkins PM: A RYR1 mutation associated with recessive congenital myopathy and dominant malignant hyperthermia in Asian families. Muscle Nerve 2009, 40:633-639.

14. Duarte ST, Oliveira J, Santos R, Pereira P, Barroso C, Conceicao I, Evangelista T: Dominant and recessive RYR1 mutations in adults with core lesions and mild muscle symptoms. Muscle Nerve 2011, 44:102-108.

15. Ducreux S, Zorzato F, Ferreiro A, Jungbluth H, Muntoni F, Monnier N, Muller CR, Treves $S$ : Functional properties of ryanodine receptors carrying three amino acid substitutions identified in patients affected by multi-minicore disease and central core disease, expressed in immortalized lymphocytes. Biochem J 2006, 395:259-266.

16. Jungbluth $H$, Davis MR, Muller C, Counsell S, Allsop J, Chattopadhyay A, Messina S, Mercuri E, Laing NG, Sewry CA, et al: Magnetic resonance imaging of muscle in congenital myopathies associated with RYR1 mutations. Neuromuscul Disord 2004, 14:785-790.

17. Jungbluth $H$, Muller CR, Halliger-Keller B, Brockington M, Brown SC, Feng L, Chattopadhyay A, Mercuri E, Manzur AY, Ferreiro A, et al: Autosomal recessive inheritance of RYR1 mutations in a congenital myopathy with cores. Neurol 2002, 59:284-287.

18. Jungbluth $\mathrm{H}$, Zhou H, Hartley L, Halliger-Keller B, Messina S, Longman C, Brockington M, Robb SA, Straub V, Voit T, et al: Minicore myopathy with ophthalmoplegia caused by mutations in the ryanodine receptor type 1 gene. Neurol 2005, 65:1930-1935.

19. Klein A, Lillis S, Munteanu I, Scoto M, Zhou H, Quinlivan R, Straub V, Manzur $A Y$, Roper $H$, Jeannet $P Y$, et al: Clinical and genetic findings in a large cohort of patients with ryanodine receptor 1 gene-associated myopathies. Hum Mutat 2012, 33:981-988.

20. Klein $A$, Jungbluth $H$, Clement $E$, Lillis $S$, Abbs $S$, Munot $P$, Pane $M$, Wraige $E$, Schara U, Straub V, et al: Muscle magnetic resonance imaging in congenital myopathies due to ryanodine receptor type 1 gene mutations. Arch Neurol 2011, 68:1171-1179.

21. Kossugue PM, Paim JF, Navarro MM, Silva HC, Pavanello RC, GurgelGiannetti J, Zatz M, Vainzof M: Central core disease due to recessive mutations in RYR1 gene: is it more common than described? Muscle Nerve 2007, 35:670-674.

22. Monnier N, Ferreiro A, Marty I, Labarre-Vila A, Mezin P, Lunardi J: A homozygous splicing mutation causing a depletion of skeletal muscle RYR1 is associated with multi-minicore disease congenital myopathy with ophthalmoplegia. Hum Mol Genet 2003, 12:1171-1178.

23. Monnier N, Laquerriere A, Marret S, Goldenberg A, Marty I, Nivoche Y, Lunardi J: First genomic rearrangement of the RYR1 gene associated with an atypical presentation of lethal neonatal hypotonia. Neuromuscul Disord 2009, 19:680-684

24. Romero NB, Monnier N, Viollet L, Cortey A, Chevallay M, Leroy JP, Lunardi J, Fardeau M: Dominant and recessive central core disease associated with RYR1 mutations and fetal akinesia. Brain 2003, 126:2341-2349. 
25. Sambuughin N, Holley H, Muldoon S, Brandom BW, de Bantel AM, Tobin JR, Nelson TE, Goldfarb LG: Screening of the entire ryanodine receptor type 1 coding region for sequence variants associated with malignant hyperthermia susceptibility in the north american population. Anesthesiol 2005, 102:515-521.

26. Schreuder LT, der Sanden MW N-v, de Hair A, Peters G, Wortmann S, Bok $L A$, Morava E: Successful use of albuterol in a patient with central core disease and mitochondrial dysfunction. J Inherit Metab Dis 2010. doi:10.1007/s10545-010-9085-7.

27. Treves S, Anderson AA, Ducreux S, Divet A, Bleunven C, Grasso C, Paesante S, Zorzato F: Ryanodine receptor 1 mutations, dysregulation of calcium homeostasis and neuromuscular disorders. Neuromuscul Disord 2005, 15:577-587.

28. Wortmann SB, Rodenburg RJ, Jonckheere A, de Vries MC, Huizing M, Heldt $K$, van den Heuvel LP, Wendel U, Kluijtmans LA, Engelke UF, et al: Biochemical and genetic analysis of 3-methylglutaconic aciduria type IV: a diagnostic strategy. Brain 2009, 132:136-146.

29. Wu S, Ibarra MC, Malicdan MC, Murayama K, Ichihara Y, Kikuchi H, Nonaka I, Noguchi S, Hayashi YK, Nishino I: Central core disease is due to RYR1 mutations in more than $90 \%$ of patients. Brain 2006, 129:1470-1480

30. Zhou $H$, Brockington M, Jungbluth $H$, Monk D, Stanier P, Sewry CA, Moore GE, Muntoni F: Epigenetic allele silencing unveils recessive RYR1 mutations in core myopathies. Am J Hum Genet 2006, 79:859-868

31. Zhou H, Lillis S, Loy RE, Ghassemi F, Rose MR, Norwood F, Mills K, AlSarraj S, Lane RJ, Feng $L$, et al: Multi-minicore disease and atypical periodic paralysis associated with novel mutations in the skeletal muscle ryanodine receptor (RYR1) gene. Neuromuscul Disord 2010, 20:166-173.

32. Zhou H, Yamaguchi N, Xu L, Wang Y, Sewry C, Jungbluth $H$, Zorzato $F$, Bertini E, Muntoni F, Meissner G, Treves S: Characterization of recessive RYR1 mutations in core myopathies. Hum Mol Genet 2006, $15: 2791-2803$

33. Zorzato $F$, Jungbluth $H$, Zhou $H$, Muntoni $F$, Treves $S$ : Functional effects of mutations identified in patients with multiminicore disease. IUBMB Life 2007, 59:14-20.

34. Bhat MB, Hayek SM, Zhao J, Zang W, Takeshima H, Wier WG, Ma J: Expression and functional characterization of the cardiac muscle ryanodine receptor $\mathrm{Ca}(2+)$ release channel in Chinese hamster ovary cells. Biophys J 1999, 77:808-816.

35. Lynch PJ, Tong J, Lehane M, Mallet A, Giblin L, Heffron JJ, Vaughan P, Zafra G, MacLennan DH, McCarthy TV: A mutation in the transmembrane/ luminal domain of the ryanodine receptor is associated with abnormal $\mathrm{Ca} 2+$ release channel function and severe central core disease. Proc Natl Acad Sci U S A 1999, 96:4164-4169.

36. Manning BM, Quane KA, Ording H, Urwyler A, Tegazzin V, Lehane $M$ O'Halloran J, Hartung E, Giblin LM, Lynch PJ, et al: Identification of novel mutations in the ryanodine-receptor gene (RYR1) in malignant hyperthermia: genotype-phenotype correlation. Am J Hum Genet 1998, 62:599-609.

37. Chamley D, Pollock NA, Stowell KM, Brown RL: Malignant hyperthermia in infancy and identification of novel RYR1 mutation. Br J Anaesth 2000, 84:500-504.

38. Monnier N, Romero NB, Lerale J, Landrieu P, Nivoche Y, Fardeau M, Lunardi J: Familial and sporadic forms of central core disease are associated with mutations in the C-terminal domain of the skeletal muscle ryanodine receptor. Hum Mol Genet 2001, 10:2581-2592.

39. Davis M, Brown R, Dickson A, Horton H, James D, Laing N, Marston R, Norgate M, Perlman D, Pollock N, Stowell K: Malignant hyperthermia associated with exercise-induced rhabdomyolysis or congenital abnormalities and a novel RYR1 mutation in New Zealand and Australian pedigrees. Br J Anaesth 2002, 88:508-515.

40. Davis KG, Wertin TM, Schriver JP: The use of simvastatin for the prevention of gallstones in the lithogenic prairie dog model. Obes Surg 2003, 13:865-868.

41. Marek KW, Davis GW: Controlling the active properties of excitable cells. Curr Opin Neurobiol 2003, 13:607-611.
42. Hirata H, Watanabe T, Hatakeyama J, Sprague SM, Saint-Amant L, Nagashima A, Cui WW, Zhou W, Kuwada JY: Zebrafish relatively relaxed mutants have a ryanodine receptor defect, show slow swimming and provide a model of multi-minicore disease. Dev 2007, 134:2771-2781.

43. Zhao M, Li P, Li X, Zhang L, Winkfein RJ, Chen SR: Molecular identification of the ryanodine receptor pore-forming segment. J Biol Chem 1999, 274:25971-25974.

44. Nelson TJ, Zhao WQ, Yuan S, Favit A, Pozzo-Miller L, Alkon DL: Calexcitin interaction with neuronal ryanodine receptors. Biochem J 1999, 341(Pt 2):423-433.

doi:10.1186/1750-1172-8-117

Cite this article as: Amburgey et al:: Genotype-phenotype correlations in recessive RYR1-related myopathies. Orphanet Journal of Rare Diseases 2013 8:117

\section{Submit your next manuscript to BioMed Central and take full advantage of:}

- Convenient online submission

- Thorough peer review

- No space constraints or color figure charges

- Immediate publication on acceptance

- Inclusion in PubMed, CAS, Scopus and Google Scholar

- Research which is freely available for redistribution

Submit your manuscript at www.biomedcentral.com/submit
C Biomed Central 